\title{
Mutability and Relationship Between Positive and Negative Symptoms During Neuroleptic Treatment in Srhizophrenia
}

\author{
Rajiv Tandon, Robert S. Goldman, JoAnn Goodson, and
} John F. Greden

\section{Introduction}

The positive/negative symptom dichotomy is being increasingly utilized to explain the heterogeneity of schizophrenia, and poor response to neuroleptic treatment has traditionally been considered to be one of the characteristic features of the negative syndrome. This issue was emphasized by Crow (1980), who principally based this conclusion on the study by Johnstone et al. (1978), which found that cis-flupenthixol, a dopamine (DA) receptor blocker, significantly reduced positive symptoms, but was no more effective on negative symptoms than trans-flupenthixol, an isomer of cis-flupenthixol without DA antagonist properties. Crow suggested that increased dopaminergic activity was the basis of the neuroleptic-responsive positive symptoms which were predominant in the so-called type I syndrome, whereas structural brain abnormalities marked by ventricular enlargement were the basis of the neuroleptic-nonresponsive negative symptoms that characterized the type II syndrome. The association between negative symptoms, poor response to neuroleptics, and ven-

From the Schizophrenia Program, University of Michigan Medical Center, Ann Arbor, $\mathrm{MI}$.

Presented at the Forty-fourth annual meeting of the Society of Biological Psychiatry, San Francisco, May 4-8, 1989.

Address reprint requests to Rajiv Tandon, M.D., Director, Schizophrenia Program, Box 0120, University of Michigan Medical Center, 1500 E. Medical Center Drive, Ann Atbor, MI 48109.

Received September 16, 1989; revised December 8, 1989. tricular enlargement was confirmed in several later studies (Andreasen et al. 1982; Angrist et al. 1980; Pearlson et al. 1984; Weinberger et al. 1980).

The National Institute of Mental Health and Veteran's Administration collaborative studies completed 25 years ago (Cole et al. 1966), however, had noted significant improvement in individual negative symptoms in schizophrenic patients as a consequence of neuroleptic treatment. This finding of improvement in negative symptoms has been confirmed in several recent studies (Breier et al. 1987; vanKammen et al. 1987; Kay and Singh 1989). One of these studies noted that improvement in negative symptoms was significantly correlated to the improvement in positive symptoms (vanKammen 1987), but another observed no such relationship (Breier et al. 1987).

In an effort to study the question of whether negative symptoms change with neuroleptic treatment and to evaluate the covariance of positive and negative symptoms, we assessed positive and negative symptoms in 40 schizophrenic inpatients at drug-free baseline and 4 weeks after neuroleptic treatment.

\section{Materials and Methods}

The sample consisted of $\mathbf{4 0}$ consecutively hospitalized patients who were admitted to the Inpatient Schizophrenia Program at the University 
of Michigan. Diagnostic evaluation included a structured interview using the Schedule of Affective Disorders and Schizophrenia (SADS) (Endicott and Spitzer 1978) as well as all available history and clinical observations. Patients had to meet both DSM-III-R (American Psychiatric fsssociation 1987) and Research Diagnostic Criteria (RDC) (Spitzer et al. 1978) criteria for schizophrenia and give informed consent for participation in the study. The sample consisted of 27 men and 13 women with a mean \pm SD age of $30 \pm 6$ years and a mean duration of illness of $6 \pm 4$ years. Twelve of the patients had never previously received any psychotropic medication, and 28 had previously received neuroleptics.

Baseline clinical ratings were performed after patients were medication free for at least 2 weeks. Patients were then placed on clinically determined doses of haloperidol or thiothixene singly, or in combination with 2-6 mg of trihexyphenidyl if they developed extrapyramidal side-effects. After about 4 weeks of neuroleptic treatment, clinical ratings were repeated. Patients were rated on the Brief Psychiatric Rating Scale (BPRS) (Overall and Gorham 1962), and the Scale for the Assessment of Negative Symptoms (SANS) (Andreasen 1983) at both timepoints. Assessment of global severity was made by the 18 -item BPRS total score. Positive symptoms were assessed by the sum of the following seven BPRS items: conceptual disorganization, mannerisms and posturing, hostility, suspiciousness, hallucinatory behavior, unusual thought content, and excitement. Negative symptoms were assessed by the SANS, with the sum of global scores being used for analysis.

Paired two-tailed $t$-tests were performed to compare the symptom ratings at baseline to those in the posttreatment phase. Correlation analysis between change in positive symptoms and change in negative symptoms was conducted to evaluate the covariance of these symptom clusters.

\section{Results}

Both positive and negative symptonis were found to improve significantly $(p<0.001)$ with neu- roleptic treatment (Table 1). Although positive symptoms improved to a greater extent than neg.ative symptoms (Figure 1), this difference was not statistically significant. Drug-naive and previously treated patients showed the same pattern of improvement (Figure 1). Use of the BPRS "THOT" factor (consisting of conceptual disorganization, suspiciousness, hallucinatory behavior, and unusual thought content) to define positive symptoms or the BPRS "ANER" factor (consisting of emotional withdrawal, motor retardation, and blunted affect) to define negative symptoms did not alter the findings.

Change in positive symptoms was significantly correlated to change in negative symptoms $(r=0.63, p<0.001)$. Even if a narrower definition of positive symptoms (the BPRS "THOT" factor) was employed, change in positive symptoms continued to be significantly correlated to change in negative symptoms $(r=0.60, p<0.001)$.

\section{Discussion}

The observed improvement in negative symptoms with neuroleptic treatment is consistent with the findings of other recent studies (Breier et al. 1987; vanKammen et al. 1987; Kay and Singh 1989) that have documented such improvement. Similar to the findings of these studies, we observed that positive symptoms improved to a somewhat greater extent than negative symptoms. The significant correlation between change in positive and negative symptoms noted in this study is in agreement with the findings of vanKammen et al. (1987), but inconsistent with Breier et al. (1987), who did not find such a relationship. The failure of Breier et al. to find this relationship may have been related to their relatively sinaller sample size ín = 19).

The covariance of positive and negative symptoms with reference to neuroleptic treatment indicates that common or related pathophysiological mechanisms may underly positive and negative symptoms in the psychotic phase of the illness. In an effort to reconcile discrepant findings with regard to neuroleptic responsiveness of negative symptoms, Meltzer (1985, in 
Table 1. Positive and Negative Symptom Ratings at Baseline and Following 4 Weeks of Neuroleptic Treatment

\begin{tabular}{lcrrrr}
\hline \multicolumn{1}{c}{ Symptom measures } & & & \multicolumn{3}{c}{ Significance } \\
\cline { 4 - 6 } \multicolumn{1}{c}{} & Baseline & Posttreatment & \multicolumn{1}{c}{$t$} & df & $p$ \\
\hline Global severity (BPRS total) & $49.3 \pm 8.0$ & $35.0 \pm 7.3$ & 11.0 & 39 & $<0.001$ \\
Positive symptoms (BPRS subscale) & $21.6 \pm 4.9$ & $13.4 \pm 4.0$ & 9.8 & 39 & $<0.001$ \\
Negative symptoms & $13.3 \pm 4.1$ & $8.7 \pm 3.7$ & 10.7 & 39 & $<0.001$ \\
(SANS sum of global scores) & & & & \\
\hline
\end{tabular}

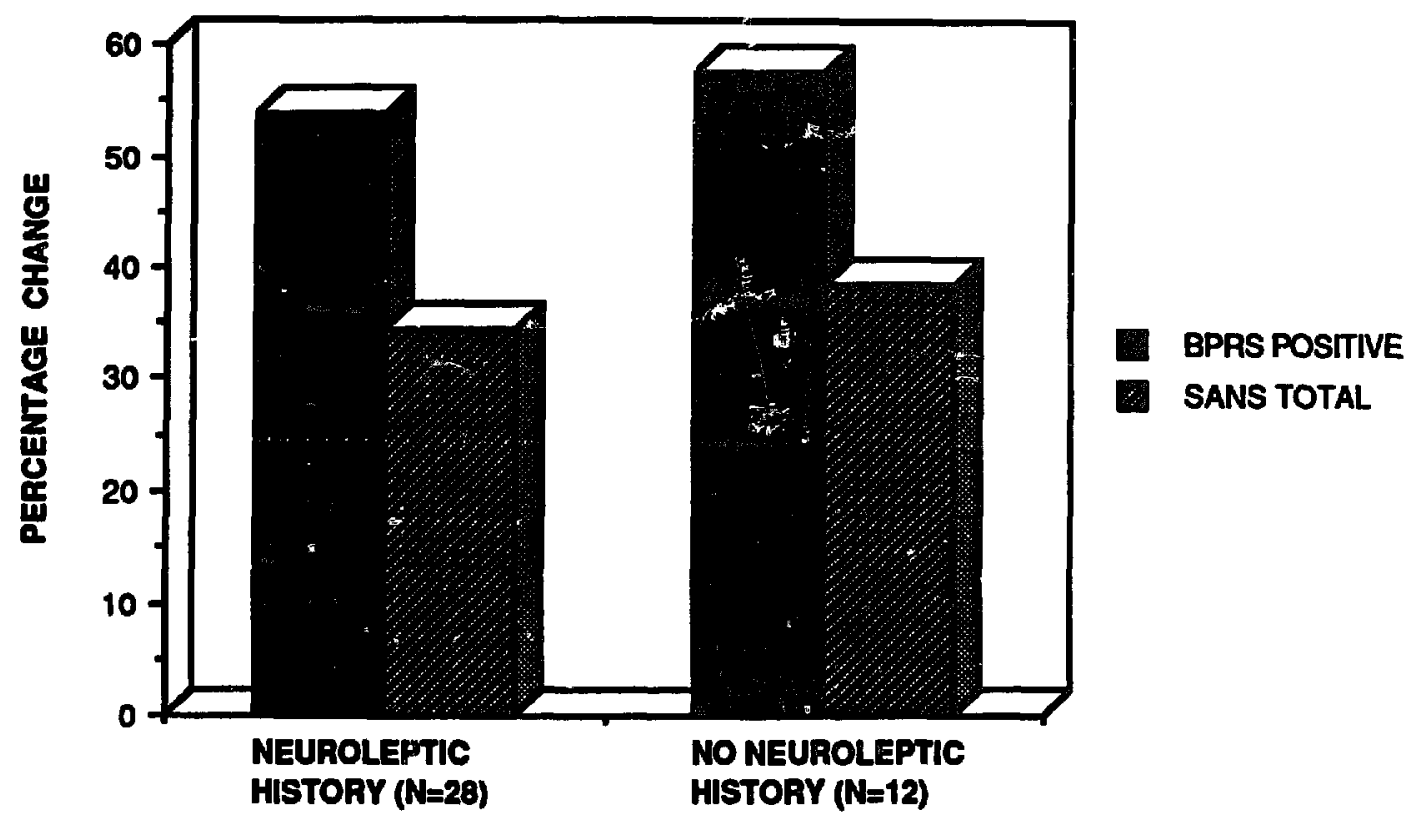

Figure 1. Degree of symptom improvement from baseline to posttreatment.

press) suggested that the effect of treatment on negative symptoms is dependent on positive symptom improvement; our findings are consistent with this contention. Alternatively, the concurrent improvement in positive and negative symptoms observed in our study is also consistent with the recently proposed model of dopaminergic/cholinergic interactions in schizophrenia (Tandon and Greden 1989) which suggests that distinct but related pathophysiological mechanisms underly positive (dopaminergic hyperactivity) and negative (muscarinic cholinergic hyperactivity) symptoms in this phase of the illness.

Though the validity of these inferences can be determined only through further study, these data are inconsistent with the characterization of negative symptoms as always being neuroleptic nonresponsive and suggest that common or related mechanisms may underly positive and negative symptoms in the psychotic phase of the illness.

\section{References}

American Psychiatric Association (1987): Diagnostic and Statistical Manual of Mental Disorders, (DSMIII-R) (ed 3). Washington DC: American Psychiatric Association.

Andreasen NC (1983): Scale for the Assessment of Negative Symptoms (SANS). Iowa City: University of Iowa.

Andreasen NC, Olsen SA, Dennert JW, Smith MR 
(1982): Ventricular enlargement in schizophrenia: Relationship to positive and negative symptoms. Am J Psychiatry 139:297-302.

Angrist B. Rotrosen J, Gershon S (1980): Differential effects of amphetamine and neuroleptics on negative vs positive symptoms in schizophrenia. Psychopharmacology 72:17-19.

Breier A, Wolkowitz OM, Doran AR, et al (1987): Neuroleptic responsivity of positive and negative symptoms in schizophrenia. Am $J$ Psychiatry 144:1549-1555.

Cole JO, Goldberg SC, Davis JM (1966): Drugs in the treatment of psychosis. In Solomon P (ed), Psycniatric Drugs. New York: Grune and Stratton, Inc, pp 153-180.

Crow TJ (1980): Molecular pathology of schizophrenia: More than one disease process. $\mathrm{Br} \mathrm{Med}$ J 280:1-9.

Endicott J, Spitzer RL (1978): A diagnostic interview: The schedule for affective disorders and schizophrenia. Arch Gen Psychiatry 35:837-844.

Johnstone EC, Crow TJ, Frith CD, Carney MWP, Price JS (1978): Mechanism of the antipsychotic effect in the treatment of acute schizophrenia. Lancet i:848-851.

Kay SR, Singh MM (1989): The positive-negative distinction in drug-free schizophrenic patients. Arch Gen Psychiatry 46:711-718.

Meltzer HY (1985): Dopamine and negative symptoms in schizophrenia: A critique of the Type I-
Type II hypothesis. In Alpert M (ed), Controversies in Schizophrenia: Changes and Constancies. New York: Guilford Press, pp 110-136.

Meltzer HY (1990): Pharmacological treatment of negative symptoms. In Greden JF, Tandon R (eds), Negative Schizophrenic Symptoms: Pathophysiology and Clinical Implications. Washington DC: American Psychiatric Press (in press).

Overall JE, Gorham DR (1962): Brief Psychiatric Rating Scale (BPRS). Psychol Rep 10:799-812.

Pearlson GD, Garbacz DJ, Breakey WR, Ahn HS, DePaulo JR (1984): Lateral ventricular enlargemert associated with persistent unemployment and negative symptoms in both schizophrenia and bipolar disorder. Psychiatry Res 12:1-9.

Spitzer RL, Endicott J, Robins E (1978): Research Diagnosti. Criteria: Rationale and reliability. Arch Gen Psychiatry 35:773-782.

Tandon R, Greden JF (1989): Cholinergic hyperactivity and negative schizophrenic symptoms. Arch Gen Psychiatry 46:745-753.

vanKanumen DP, Hommer DW, Malas KL (1987): Effect of pimozide on positive and negative symptoms in schizophrenic patients: Are negative symptoms state dependent? Neuropsychobiology 18:113-117.

Weinberger DR, Bigelow LB, Kleinman JE, Klein JT, Rosenblatt JE, Wyatt RJ (1980): Cerebral ventricular enlargement in chronic schizophrenia: An association with poor response to treatment. Arch Gen Psychiatry 37:11-13. 